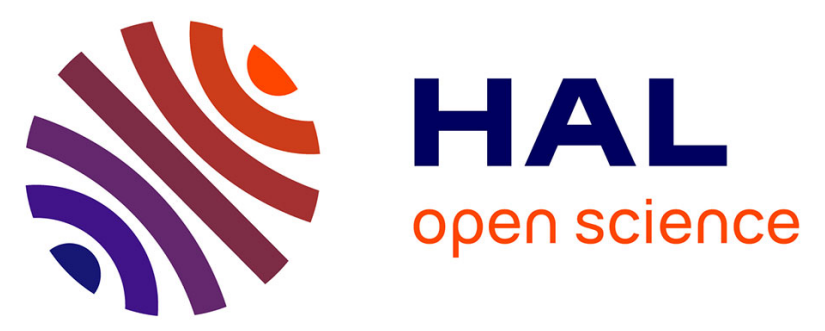

\title{
A DFT-based model for water adsorption at aluminosilicate surfaces. Comparison with experimental data extracted from dielectric relaxation spectroscopy
}

Habib Belarbi, Ahmed Haouzi, Jean-Charles Giuntini, Sabine

Devautour-Vinot, Mohamed Kharroubi, François Henn

\section{To cite this version:}

Habib Belarbi, Ahmed Haouzi, Jean-Charles Giuntini, Sabine Devautour-Vinot, Mohamed Kharroubi, et al.. A DFT-based model for water adsorption at aluminosilicate surfaces. Comparison with experimental data extracted from dielectric relaxation spectroscopy. Journal of Non-Crystalline Solids, 2010, 356, pp.664. 10.1016/j.jnoncrysol.2009.09.040 . hal-00584844

\section{HAL Id: hal-00584844 \\ https://hal.science/hal-00584844}

Submitted on 19 May 2011

HAL is a multi-disciplinary open access archive for the deposit and dissemination of scientific research documents, whether they are published or not. The documents may come from teaching and research institutions in France or abroad, or from public or private research centers.
L'archive ouverte pluridisciplinaire HAL, est destinée au dépôt et à la diffusion de documents scientifiques de niveau recherche, publiés ou non, émanant des établissements d'enseignement et de recherche français ou étrangers, des laboratoires publics ou privés. 


\title{
A DFT-based model for water adsorption at aluminosilicate surfaces.
}

\section{Comparison with experimental data extracted from dielectric relaxation spectroscopy.}

\author{
$\underline{\text { H. Belarbi }}{ }^{1, *}$, A. Haouzi ${ }^{1}$, J.C. Giuntini ${ }^{1}$, S. Devautour-Vinot ${ }^{2}$, M. Kharroubi ${ }^{2}$ \& F. Henn $^{2}$
}

\author{
${ }^{1}$ Laboratoire Synthèse et Catalyse, Université de Tiaret, 14000 Tiaret, Algeria \\ ${ }^{2}$ Equipe Physico-chimie des Matériaux Désordonnés et Poreux, Institut Charles Gerhardt de \\ Montpellier (UMR 5253), Université Montpellier 2, Place Eugène Bataillon, 34095 \\ Montpellier cedex 5, France \\ * Corresponding author: belarbi@mail.univ-tiaret.dz
}

\begin{abstract}
When applied to adsorption phenomena, Gibbs-Duhem equation shows that surface energy associated to exchangeable cations located at the aluminosilicate surface decreases with the increasing number of adsorbed molecules. In this work, we propose a microscopic representation of this phenomenon, i.e. the adsorption of water molecules at the cationic sites, and its evolution upon water adsorption. Accordingly, the DFT-based model proposed in this paper provides a simple mathematical expression in which the system, i.e. cation/surface, energy is a functional of the electronic density. Therefore, the adsorption of small molecules as water which results in modification of the surface electronic density and, hence, of the electrical potential modifies the barrier for cation hopping. The model presented here is favourably confronted to experimental data of energy barrier for cation hopping measured by
\end{abstract}


dielectric relaxation, i.e. complex impedance spectroscopy and thermally stimulated current, on various aluminosilicate solids: two zeolites (Na-Faujasite and Na-Mordenite) and clay mineral (Na-Montmorillonite) upon water adsorption.

\section{Introduction}

\section{Theory}

\section{I- Gibbs Duhem's law}

In the past, only phenomenological and over simplified approaches could provide exploitable information on the properties of solid surfaces. During the last decades, scientists have, however, developed models to explain the microscopic aspect of the surface properties. This achievement was obviously explained by an increase request for more accurate estimation of the quantitative experimental parameters controlling the microscopic mechanisms of adsorption. In that context, we showed in recent papers $[1,2]$ that measurements of dielectric relaxation spectroscopy, i.e. impedance spectroscopy and thermally stimulated currents, could be used to evaluate energies of interactions that exist on the surface of solids where cations are trapped.

Gibbs [3] was the first to notice that the necessary work to create a unit of surface on a solid was different according to the nature of the stresses exerted on its structure. Basically, it is important to use a tensor to determine the response to these stresses. D.H. Everett and P.R. Couchmann $[4,5]$ explained how to circumvent this difficulty and proposed to create the parameter: $\gamma_{s}$ called: "the generalized surface energy" that is related to the elastic strain at the surface. In this work, providing that the localized hops of the cations trapped at the aluminosilicate surface are associated with dipolar polarization, we assume it corresponds to a typical example of surface deformation. 
From the symbol originally used by J.W. Gibbs, the surface concentration of a given component $i$, is noted $\Gamma_{i}$ and defined as:

$$
\Gamma_{i}=\frac{n_{i}}{A}
$$

Where $A$ is the area of the considered surface and $n_{i}$ the number of molecules of the component $i$ adsorbed on the surface of the solid.

In a solid, the elementary deformation $d A$, cannot generally be compensated by a migration of atoms. This property clearly distinguishes the variations of the surface of solids from those of liquids. However, when the applied stress is weak, "elastic" deformation can be observed. The model proposed here assumed that the hopping of cations observed on a microscopic scale yields this type of deformation and that the presence of adsorbed molecules in the cation vicinity will necessarily modify the cation hopping barrier and hence the surface energy.

We can write the differential $d \sigma$ of the work of the solid surface, during the transformation considered, in the form:

$$
d \sigma=\gamma_{s} d A
$$

The relation of Gibbs Duhem allows us to evaluate this differential work during an isothermal transformation. To carry out this calculation, we must initially define the surface work as the reversible work necessary to create a unit of surface $d A$, when the temperature, the electric field, the chemical potential and the elastic stresses are maintained constant.

$\gamma_{s}$ can then be defined according to the "surface work": $\sigma$ and the "surface stresses", characterized by the tensor Y [3]:

$$
\gamma_{s}=\frac{d \varepsilon_{p}}{d \varepsilon_{t o t}} \sigma+\frac{d \varepsilon_{e}}{d \varepsilon_{t o t}} \mathrm{Y}
$$


Where $d \varepsilon_{\text {tot }}$ is the total surface strain, $d \varepsilon_{e}$ the elastic surface strain and $d \varepsilon_{p}$ the plastic surface strain.

When applied to a surface system, such as assumed in this paper, it leads to Gibbs-Duhem's law for the surface separating the gas from the solid phase. To reach that goal, we must define the surface entropy, $s_{s}$, as the variation of the surface energy caused by an increase in temperature whilst all other parameters are constant. At equilibrium, we then obtain [3]:

$$
s_{s} d T+\sum_{i} \Gamma_{i} d \mu_{i}+q_{e s} d E+\left(\sigma-\gamma_{s}\right) d \varepsilon_{e}+d \sigma=0
$$

When the evolution of the system is at a constant temperature, constant electric field, $(d E=0)$ and no plastic strain $\left(d \varepsilon_{p}=0\right)$, the Gibbs-Duhem's equation makes possible to write $\Gamma_{i}$ :

$$
\Gamma_{i}=\left(-\frac{\partial \sigma}{\partial \mu_{i}}\right)_{\mu_{k \neq i}, T, E, \varepsilon_{e}}
$$

Where, in that case, $i$ indicates the considered component in the vapor phase and $\mathrm{k}$ all the other components in the same phase. If gas $i$ is regarded as a perfect gas, it is possible to write:

$$
d \mu_{i}=R T d\left[\ln \left(p_{i}\right)\right]=R T \frac{d p_{i}}{p_{i}}
$$

so that eq. (5) yields:

$$
\frac{R T \Gamma_{i}}{p_{i}}=-\left(\frac{\partial \sigma}{\partial p_{i}}\right)
$$


It must be outlined that eq.(7) shows that the higher the number of adsorbed molecules, the less important is surface energy decrease. That means that upon adsorption, the variation of work that is developed at the surface becomes smaller and smaller. Consequently, this trend should also be obeyed by the energy barrier for cation hopping.

\section{II- A simple DFT-based model for the electronic cloud energy of a cation confined in a surface site.}

The phenomenon of dielectric relaxation observed in aluminosilicates can be associated to the localized hopping motion of the cations trapped on the surface [6]. Therefore, one way to confront the microscopic description given above to experimental data, is to compare the values of the energy barrier associated to the cation hopping, $\Delta E$, that can be extracted from dielectric relaxation experiments with the model prediction. Our objective thus consists in obtaining an analytical expression for $\Delta E$. For that purpose, DFT-based concepts are used.

Assuming the variation of electronic cloud energy of a species that belongs to the studied surface can be expressed by a series of second order of two variables: (i) $\delta N$ the variation of the number of the electrons that constitute the electronic cloud of the system and (ii) $\delta V_{s}$ the modification of the electric potential, that surrounds the system, it comes:

$$
E=E_{0}+\left(\frac{\partial E}{\partial N}\right)_{V_{s}} \delta N+\left(\frac{\partial E}{\partial V_{s}}\right) \delta V_{s}+\frac{1}{2}\left(\frac{\partial^{2} E}{\partial N^{2}}\right)(\delta N)^{2}+\frac{1}{2}\left(\frac{\partial^{2} E}{\partial V_{s}^{2}}\right)\left(\delta V_{s}\right)^{2}+\left(\frac{\partial^{2} E}{\partial N \partial V_{s}}\right) \partial N \partial V_{s}
$$

By definition: $\left(\frac{\partial E}{\partial V_{s}}\right)=\rho$ is the electronic density, $\left(\frac{\partial E}{\partial N}\right)_{V_{s}}=\mu$ the chemical potential of the electrons and $\left(\frac{\partial^{2} E}{\partial N^{2}}\right)_{V_{s}}=\eta$ the chemical hardness of the considered system. 
Following the developments suggested by J.W. Mortier [7] on the one hand and, Parr and Yang [8] on the other hand, we obtain the energy barrier for cation hopping:

$$
E_{c}=\mu_{0, c} \delta N+\int_{0}^{\infty} \rho_{c} \delta V_{s} d r+\eta_{c}(\delta N)^{2}+\delta N \int_{0}^{\infty} f_{c}(r) \delta V_{s} d r+\iint\left(\frac{\partial \rho_{c}}{\delta V_{s}}\right) \delta V_{s}(r) \delta V_{s}\left(r^{\prime}\right) d r d r^{\prime}
$$

Where $f$, the Fukui function, is equal to $\left(\frac{\partial \rho}{\partial N}\right)_{V_{s}}$ and the subscript $c$ corresponds to the cation.

Using the same equation for the aluminosilicate surface surrounding the cation and assuming that the energy barrier corresponds to the energy difference between the energy of the cation trapped at the surface and that when it is free, i.e. detrapped, we can write:

$$
\begin{aligned}
& \Delta E=\left(\mu_{0, c}-\mu_{0, s}\right) \delta N+\int_{0}^{\infty} \rho_{c} \delta V_{s} d r+\int_{0}^{\infty} \rho_{s} \delta V_{c} d r+\cdots \\
& \cdots+\left(\eta_{c}+\eta_{s}\right)(\delta N)^{2}+\delta N\left[\int_{0}^{\infty} f_{c} \delta V_{s} d r+\int_{0}^{\infty} f_{s} \delta V_{c} d r\right]+\cdots \\
& \cdots+\iint\left(\frac{\partial \rho_{c}}{\delta V_{s}}\right) \delta V_{s}(r) \delta V_{s}\left(r^{\prime}\right) d r d r^{\prime}+\iint\left(\frac{\partial \rho_{s}}{\delta V_{c}}\right) \delta V_{c}(r) \delta V_{c}\left(r^{\prime}\right) d r d r^{\prime}
\end{aligned}
$$

Eq. (10), first found by Parr and Yang [7], correspond to the sum of three terms that can be identified as the three energetic contributions, i.e. electrostatic, covalency and polarisation, involved in a chemical bond:

$$
\Delta E=\Delta E_{\text {elec }}+\Delta E_{\mathrm{cov}}+\Delta E_{\text {pol }}
$$

We then tried to apply this type of calculation to the determination of the variation of the energy of hopping, $\Delta E$, that can be measured by means of dielectric relaxation spectroscopy in the dehydrated state as well as a function of the number of adsorbed molecules. For that purpose, the following assumptions have been proposed:

i) the cation electronic density and the cation polarisability do not change during the hopping process. This implies that $\delta \rho_{c}=0$ and $\delta V_{c}=0$. 
ii) for the first stage of adsorption, the cation is not fully solvated by the adsorbed molecule, i.e. there is no adsorbed molecule between the cation and the surface.

iii) the energy variation can be correctly, at least for a qualitative investigation, limited to a first order approximation.

As a consequence, eq.10 becomes:

$$
\delta(\Delta E)=\left(\mu_{0, c}-\mu_{0, s}\right) \delta N+\int_{r_{0}}^{r} \rho_{c} \delta V_{s} d r
$$

The integral boundaries are $r_{o}$ which represents the cationic site radius and $r$ the cut-off radius beyond which the adsorption of a molecule does not significantly influence the hopping barrier for the cation.

Upon adsorption, $\mu_{0, s}$, the chemical potential of the surface and of the adsorbed molecules surrounding the cationic site, $\delta N$, the variation of electronic charges and $\delta V_{s}$, the variation of the electrical potential exerted on the cation will change accordingly to the nature of the adsorbed species $i$ and to its surface concentration $\Gamma_{i}$.

We now introduce $\delta N_{i}$ the contribution of the adsorbed species $i$ to the number of electronic charge at the surface and $B$ a parameter that accounts for the stœchiometric ratio between the dry surface and the number of adsorbed molecules by area unit. In other terms, $B$ represents the ratio between the number of atoms at the surface that constitutes an adsorption site and the number of atoms of the adsorbed species.

We aim now at expressing eq.12 as a function of the number of adsorbed species. Assuming that only one type of species $i$ is adsorbed, we can write the variation of electronic charges, $\delta N$, simply results from the concentration of species $i$ :

$$
\delta N=\delta N_{i} \Gamma_{i}
$$

Noteworthy, $\delta N_{i}$ can be fractional and lower than one. 
By definition, the transfer of electronic charge goes from the less to the more electroactive species. It can then be shown from DFT that the number of electronic charges that will be exchanged between the surface and the adsorbed species is:

$$
\delta N_{i}=\frac{\chi_{s}-\chi_{i}}{2 \alpha\left(\eta_{s}+\eta_{i}\right)}
$$

where $\alpha$ is an empirical parameter and $\chi$ the electronegativity that is opposite to the chemical potential, i.e. $\chi=-\mu . \quad \chi_{s}$ can be calculated using the group electronegativity concept developed by Sanderson [9] providing that the electronegativity of the aluminosilicate $\chi_{a}$ and of the adsorbed species $\chi_{i}$ are known:

$$
\chi_{s}=\left(\chi_{a}\right)^{\frac{B}{B+\Gamma_{i}}} \cdot\left(\chi_{i}\right)^{\frac{\Gamma_{i}}{B+\Gamma_{i}}}
$$

Let us now define $A_{i}$ as the area occupied by an adsorbed molecule of species $i$. The maximum number of absorbed molecules contained in a crown centered around a given cation site can thus be expressed by:

$$
\Gamma_{i}=\pi\left(\frac{r^{2}-r_{0}^{2}}{A_{i}}\right)
$$

where $r$ and $r_{0}$ are the external and internal radius of the crown respectively. $r_{0}$ can be seen as the minimum distance separating the cation and the adsorbed molecule.

Therefore, providing $\rho_{c}$ is constant and $\delta V_{s}$ is due to the modification of the charges, caused by the adsorption of the molecules, the term $\int_{r_{0}}^{r} \rho_{c} \delta V_{s} d r$ in eq. (12) becomes:

$$
\int_{r_{0}}^{r} \rho_{c} \delta V{ }_{s} d r=\rho_{c} \int_{r_{0}}^{r} \delta N_{i} \Gamma_{i} \frac{d r}{r}=\delta N_{i} \pi \rho_{c} \int_{r_{0}}^{r}\left(\frac{r^{2}-r_{0}^{2}}{A_{i}}\right) \frac{d r}{r}
$$

and, after integration,: 


$$
\int_{r_{0}}^{r} \rho_{c} \delta V_{s} d r=\frac{\pi \rho_{c} \delta N_{i}}{A_{i}}\left[\left(\frac{r^{2}-r_{0}^{2}}{2}\right)-r_{0}^{2} \ln \left(\frac{r}{r_{0}}\right)\right]
$$

The combination of eqs (16) and (18) leads to:

$$
\int_{r_{0}}^{r} \rho_{c} \delta V_{s} d r=\delta N_{i} \rho_{c}\left[\left(\frac{\Gamma_{i}}{2}\right)-\frac{\pi r_{0}^{2}}{A_{i}} \ln \left(\sqrt{\frac{A_{i} \Gamma_{i}}{2 \pi r_{0}^{2}}+1}\right)\right]
$$

We can now express the evolution of the energy barrier as the function of $\Gamma_{i}$, the concentration of adsorbed molecules in the cation vicinity (eqs. $12,1314 \& 20$ ):

$$
\delta(\Delta E)=\left[\begin{array}{c}
\frac{\left.\left(\chi_{a}\right)^{\frac{B}{B+\Gamma_{i}}}\left(\chi_{i}\right)^{\frac{\Gamma_{i}}{B+\Gamma_{i}}}\right)-\chi_{i}}{2 \alpha\left(\eta_{a}+\eta_{i}\right)} . \\
\left.\chi_{0, c}-\left(\chi_{a}\right)^{\frac{B}{B+\Gamma_{i}}}\left(\chi_{i}\right)^{\frac{\Gamma_{i}}{B+\Gamma_{i}}}\right)+\ldots \\
\ldots+\rho_{c}\left[\left(\frac{1}{2}\right)-\frac{\pi r_{0}^{2}}{A_{i} \Gamma_{i}} \ln \left(\sqrt{\frac{A_{i} \Gamma_{i}}{\pi r_{0}^{2}}+1}\right)\right]
\end{array}\right] \Gamma_{i}
$$

Electronegativity $\chi_{i}$ and chemical hardness $\eta_{i}$ of the adsorbed molecules, are evaluated by using the Allen'scale $[9,10]$ and the PACHA code [11], see table 1.

If the exchanged cation is $\mathrm{Na}^{+}$then $r_{0}=1,1310^{-10} \mathrm{~m}$ and if the adsorbed molecule is water then $A_{i}=1310^{-20} \mathrm{~m}^{2}$.

\section{Materials and methods}

The experimental data confronted to the model predictions have been collected from Dielectric Relaxation Spectroscopy experiments carried out on two zeolites, i.e. a mordenite and a faujasite $\mathrm{Y}$, and a swelling clay mineral, i.e. a montmorillonite. Each material has been 
completely exchanged with sodium, i.e. the measured energy barrier can be associated to the hopping of sodium cation. Their chemical formula are given by:

- $\quad$ Mordenite [13]: $\mathrm{Na}_{7.4} \mathrm{Si}_{40.6} \mathrm{Al}_{7.4} \mathrm{O}_{96}, \mathrm{nH}_{2} \mathrm{O}$ (Si/Al: 5.5).

- Faujasite $\mathrm{Na}-\mathrm{Y}$ [12]: $\mathrm{Na}_{56} \mathrm{Si}_{136} \mathrm{Al}_{56} \mathrm{O}_{384} \mathrm{nH}_{2} \mathrm{O}$ (Si/Al: 2.4).

- Montmorillonite [14]: $\left(\mathrm{Si}_{4.2}\right)_{\text {IV }}\left(\mathrm{Al}_{1.2} \mathrm{Mg}_{0.2} \mathrm{Fe}_{0.2}\right)_{\mathrm{VI}}(\mathrm{OH})_{2}, \mathrm{nH}_{2} \mathrm{O}, \mathrm{Na}_{0.1}, \mathrm{~K}_{0.1}$

where the subscripts IV and VI indicate the tetrahedral and octahedral layers in the clay sheet. All these data as well as the corresponding experimental procedures have already been published [1, 2,12-14].

To make it short, dielectric spectra were measured on pellets made of compressed power using a BDS-4000 Novocontrol spectrometer coupled to the Quatro system which insures the temperature control. The activation barrier, $\Delta E$, is calculated from the temperature dependence of the mean relaxation $\tau$ associated to the mean energy barrier via:

$$
\tau=\tau_{0} \exp \left(\frac{\Delta E}{k_{B} T}\right)
$$

The determination of $\Delta E$ at various water loadings, that are proportional to $\Gamma_{\mathrm{i}}$, was obtained according to the following procedure. The aluminosilicate pellet was initially placed in a saturated water vapor atmosphere for $24 \mathrm{~h}$ in order to be fully hydrated. Using thermogravimetric analysis (TGA), the sample was heated at several constant Treatment Temperatures (TT) until equilibrium condition was reached, i.e. $2 \mathrm{~h}$. The departure of water molecules per cation, $\mathrm{nH}_{2} \mathrm{O}$, was measured for each TT from weight loss providing the weight loss measured only corresponds to water loss. The exact same thermal conditions were then reproduced in the dielectric spectrometer cell, and once $\mathrm{nH}_{2} \mathrm{O}$ was equilibrated, $\varepsilon "(\omega)$ vs $\omega$ spectra were recorded at various fixed temperatures lower than TT. This procesure ensures that the water molecule loading, $\mathrm{nH}_{2} \mathrm{O}$, remains constant throughout the experiment. $\Delta E$ can thus be determined for each constant $\mathrm{nH}_{2} \mathrm{O}$ value. It must be emphasized that $\mathrm{Eq}(22)$ is 
obeyed for all samples and for each water loading. This result proves that $\mathrm{nH}_{2} \mathrm{O}$ is effectively constant for a set of temperatures of measurements below TT.

\section{Results}

On figures 1,2 and 3, we compare the values of $\Delta E$ obtained from eq.(21) with those obtained from dielectric relaxation measurements. The fitting procedure only leaves two adjustable parameters: $\alpha$ and $B$. It appears clearly that the theoretical expression described by eq.(21) reproduces satisfactorily the experimental behaviors observed on the three studied materials. This outcome shows that DRS measurements on these types of aluminosilicate solids subjected to water adsorption, makes possible to follow the evolution of the surface energy of these materials in particular points of the surface, i.e. cations site. We can, thus, consider that DRS is a fruitful tool for investigating cationic surfaces upon adsorption of water.

The values of the parameters $\alpha$ and $B$ (eq.21) gained from the fitting of the experimental data are reported in table 1 . On the one hand, $\alpha$ is a parameter that characterizes the interaction between the adsorbed molecule and the aluminosilicate surface (see eq. 14) of studied samples. In other words, it is associated to the capability of both the surface and the adsorbed molecule to exchange electrons. The data reported in table 1 show that $\alpha$ is almost the same for the three samples investigated here. This results is not astonishing since the chemical nature of the three investigated materials is very similar. In all cases, the surface is made of oxygen atoms bearing negative charges and of sodium cations. On the other hand, the parameter $B$ gives an account of the stoechiometric coefficient that relates the number of atoms of the dry aluminosilicate surface over the number of water molecules per unit of area. Therefore, the larger is $B$, the larger, i.e. the less localized, the size of the adsorption site. The fitting of the experimental data clearly demonstrates (see table 1) that the value of $B$ for 
the two zeolites can be distinguished from that of the montmorillonite. This outcome is expected since these two types of materials do not have the same specific BET area (see table 1) nor the same density of $\mathrm{Na}^{+}$per $\mathrm{Si}$ atoms (see chemical formula given in the Material and Method section). It is then remarkable that the lower the density of $\mathrm{Na}^{+}$cations the higher the parameter $B$ as expected from its definition. Consequently, it must be outlined that both the qualitative evolutions of $B$ and $\alpha$ match well the theoretical expectations.

\section{Conclusion}

DRS experiments carried out on three typical aluminosilicates having $\mathrm{Na}^{+}$cations at their surface, reveal that the evolution of barrier energy for cation hopping according to the number of adsorbed water molecules follows the phenomenological Gibbs-Duhem's law. Furthermore, a microscopic model based on DFT principles is proposed and favourably compared to the experimental data extracted from DRS. The parameters that are extracted from the fitting of the experimental evolutions with the equation derived from the model shows that the charge density of the network is the key factor. This outcome is, of course, not surprising. However, it shows that the simple microscopic model proposed is, at least for the materials studied here, acceptable. It also emphazises that DRS can be a convenient tools for investigating surface properties and adsorption mechanisms when the cations located at the surface correspond to the adsorption sites. It is likely that similar conclusions could be obtained when the adsorbing surface is made of "fixed" dipoles as in many polymers. 


\section{References}

[1] J.C. Giuntini, J.M. Douillard, G. Maurin, S. Devautour-Vinot, A. Nicolas, F. Henn, Chem. Phys. Let. 423 (2006) 71.

[2] H. Belarbi, A. Haouzi, J.M. Douillard, J.C. Giuntini, F. Henn, J. Colloid Interface. Sci. 308 (2007) 216.

[3] R. G. Linford, Chem. Rev. 78 (1978) 81.

[4] D.H. Everett, P.R. Couchman, J. Colloid lnterface Sci. 52 (1975) 410.

[5] P.R. Couchman, D.H. Everett, J. Electroanal. Chem. Interfacial Electrochem. 67 (1976) 382.

[6] G. Maurin, P. Senet, S. Devautour, F. Henn, J.C. Giuntini, V.E. Van Doren, Comp. Mater. Sci. 22 (2001) 106.

[7] W.J. Mortier, Compilation of Extraframework Sites in Zeolites, Butterworth Scientific Ltd., Guildford, 1982.

[8] R.G. Parr, W. Yang, Density-Functional Theory of Atoms and Molecules, International Series of Monographs on Chemistry, Oxford Univ. Press, New York, 1994.

[9] J.E. Huheey, E.A. Keiter, R.L. Keiter, Inorganic Chemistry: Principles of Structure and Reactivity, $4^{\text {th }}$ ed., HarperCollins, New York, 1993.

[10] L.C. Allen, J. am. Chem. Soc. 111 (1989) 9003.

[11] M. Henry, Chem. Phys. Chem. 3 (2002) 561.

[12] A. Nicolas, S. Devautour-Vinot, G. Maurin, J.C. Giuntini, F. Henn, Microporous and Mesoporous Materials 109 (2008) 413.

[13] J.M. Douillard, G. Maurin, F. Henn, S. Devautour-Vinot, J.C. Giuntini J. Colloid Interface Sci. 306 (2007) 440.

[14] V. Médout-Marère, H. Belarbi, P. Thomas, F. Morato, J.C. Giuntini, J. Mr. Douillard, J. Colloid Interface Sci. 202 (1998) 139. 
[15] J. Llorens \& M. Pera-Titus, J. Colloid Interface Sci. 331 (2009) 302. 


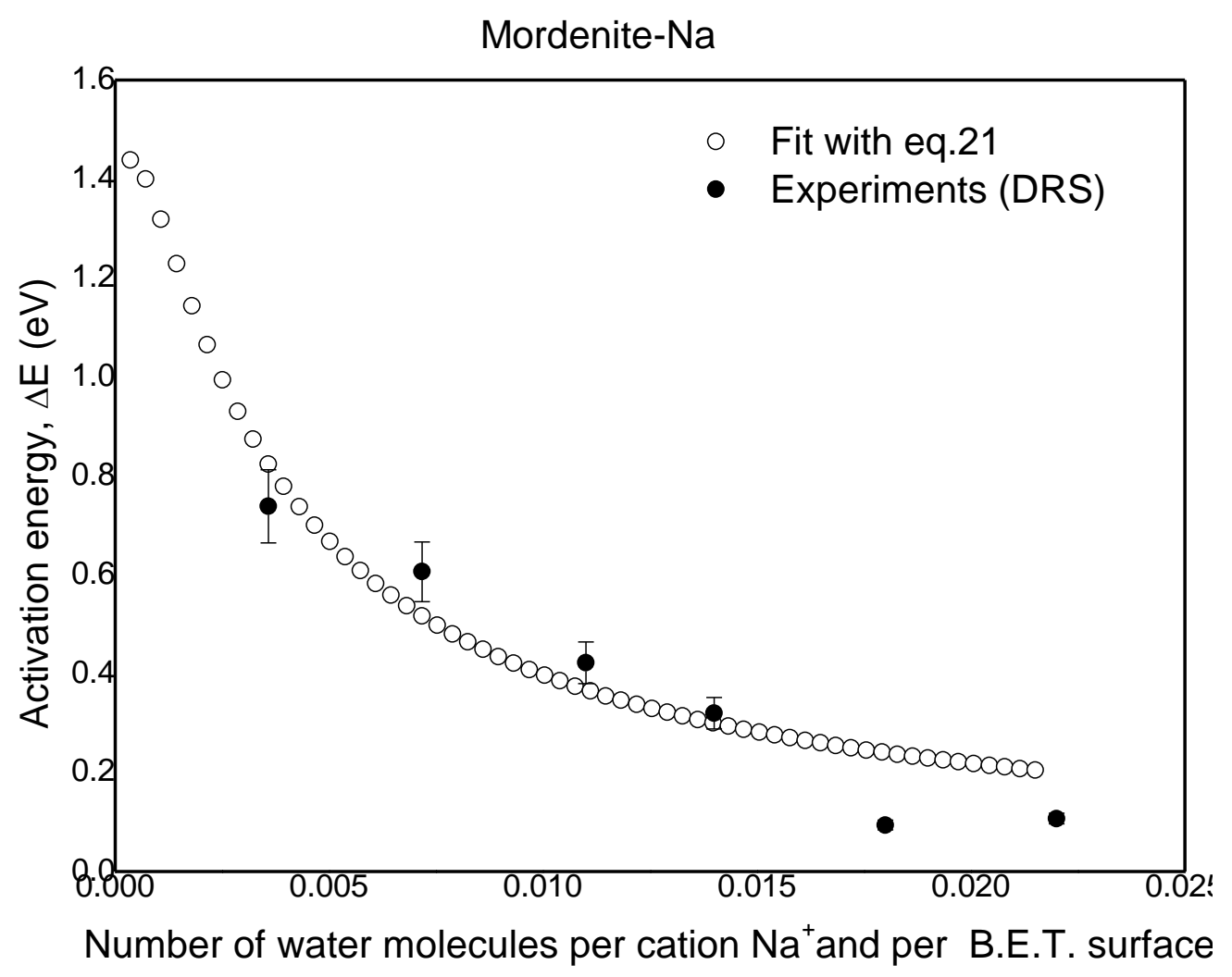

Figure 1. 
Faujasite $\mathrm{Na}-\mathrm{Y}$

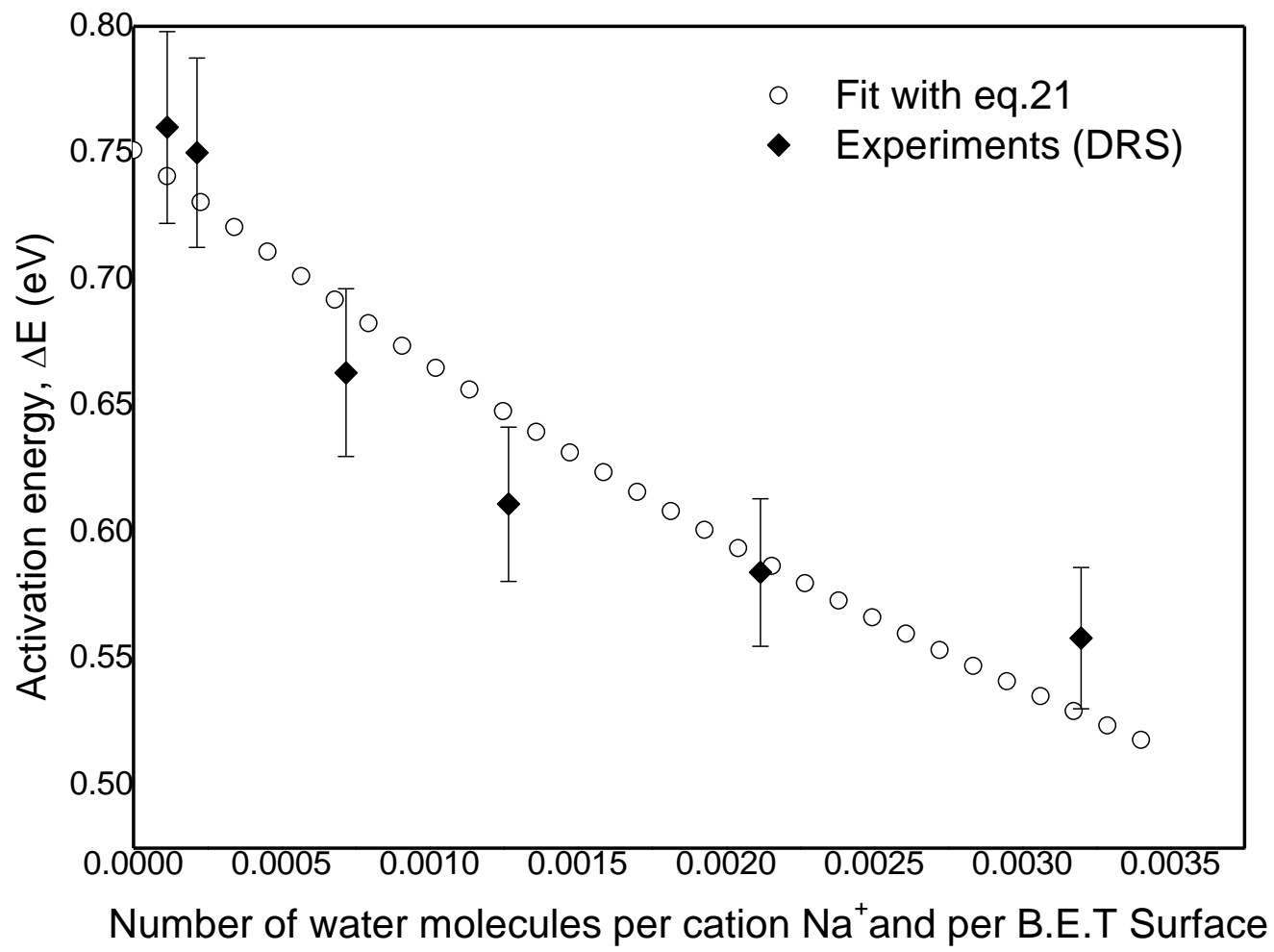

Figure 2. 


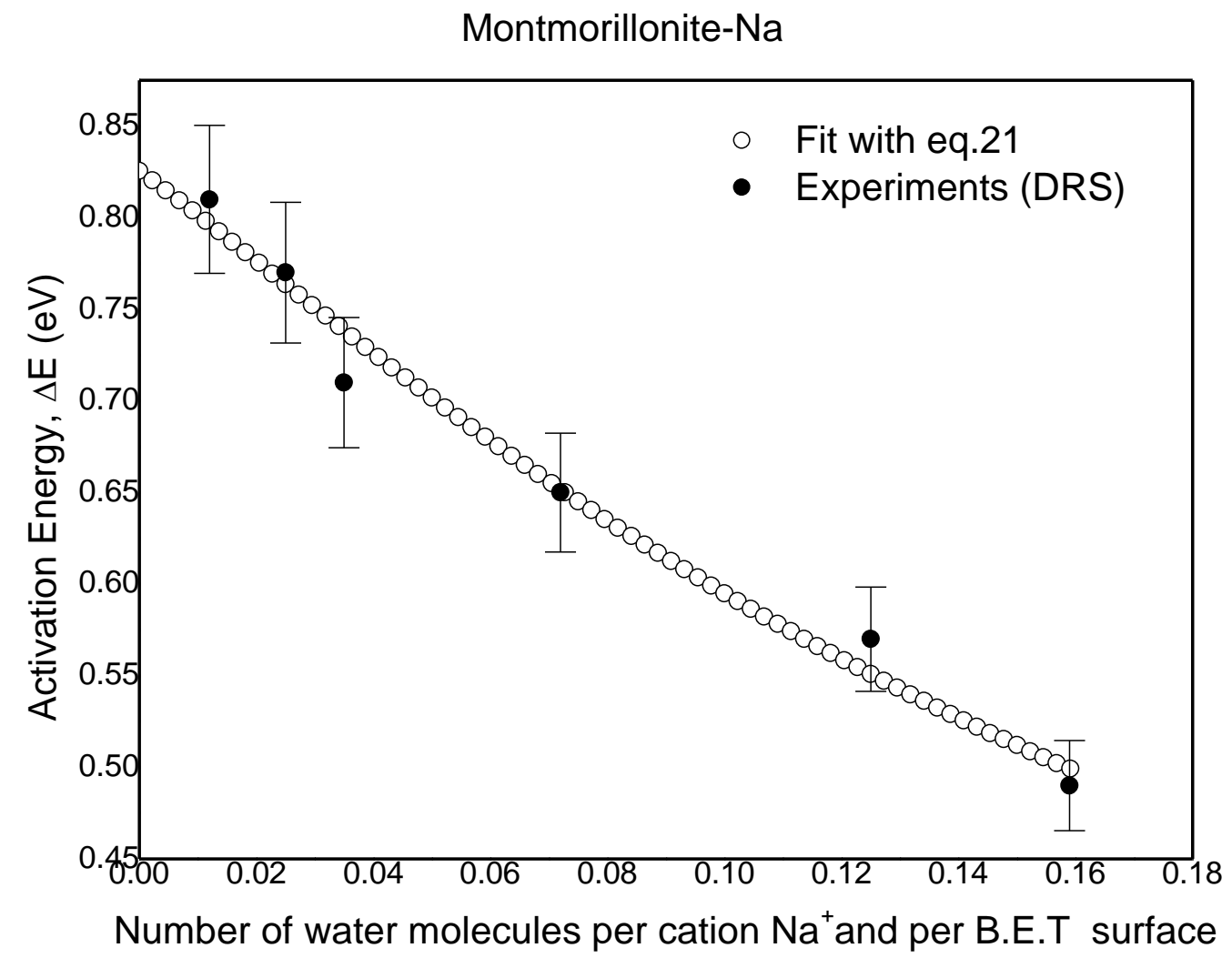

Figure 3. 
Figure captions

Figure 1: Variation of the energy barrier for cation hopping measured from dielectric relaxation measured versus the number of adsorbed water molecules per $\mathrm{Na}^{+}$and per $\mathrm{BET}$ surface. Comparison between experimental data and theoretical data calculated with eq. 21 . Case of Na-mordenite[13].

Figure 2: Variation of the energy barrier for cation hopping measured from dielectric relaxation measured according to the number of adsorbed water molecules per $\mathrm{Na}^{+}$and per BET surface. Comparison between experimental data and theoretical data calculated with eq. 21. Case of Faujasite-NaY.

Figure 3: Variation of the energy barrier for cation hopping measured from dielectric relaxation measured according to the number of adsorbed water molecules per $\mathrm{Na}^{+}$and per BET surface. Comparison between experimental data and theoretical data calculated with eq. 21. Case of Na-montmorillonite[2]. 


\begin{tabular}{|c|c|c|c|c|c|c|}
\hline Aluminosilicate & $\begin{array}{c}\text { B.E.T surface } \\
\left(\mathrm{m}^{2} / \mathrm{g}\right)\end{array}$ & $\chi$ & $\eta$ & $\alpha$ & B & $\mathrm{R}$ (coef. corr.) \\
\hline $\begin{array}{l}\text { Mordenite } \\
\text { (Si/Al=5.5) }\end{array}$ & 279 [15] & 9.93 & 3.04 & 0.39 & $1.3610^{-3}$ & 0.90 \\
\hline $\begin{array}{c}\text { Faujasite })-\mathrm{NaY} \\
\quad(\mathrm{Si} / \mathrm{Al}=2.4)\end{array}$ & 882 [15] & 12.22 & 4.02 & 0.57 & $4.6210^{-3}$ & 0.97 \\
\hline Montmorillonite & $44[14]$ & 10.99 & 1.64 & 0.71 & 0.11 & 0.98 \\
\hline \multicolumn{3}{|c|}{ Cation: Sodium } & \multicolumn{4}{|c|}{ Solvent: water } \\
\hline \multicolumn{3}{|c|}{$\begin{array}{l}\chi_{c}=5.14 \\
\eta_{c}=8.4\end{array}$} & \multicolumn{4}{|c|}{$\begin{array}{l}\chi_{i}=14.87 \\
\eta_{i}=17.80\end{array}$} \\
\hline
\end{tabular}

Table 1: Calculated values of parameters $\alpha$ and $B$ extracted from the fitting of experimental data with eq. 21 (see figs. 1, 2 and 3) 